\title{
Safety of Eptifibatide for Subcortical Stroke Progression
}

\author{
Sheryl Martin-Schild Hashem Shaltoni Anitha T. Abraham Andrew D. Barreto \\ Hen Hallevi Nicole R. Gonzales James C. Grotta Sean I. Savitz \\ Department of Neurology, University of Texas-Houston Medical School, Houston, Tex., USA
}

\section{Key Words}

Stroke $\cdot$ Subcortical infarct Eptifibatide

\begin{abstract}
Background: There is no proven treatment for stroke progression in patients with subcortical infarcts. Eptifibatide, a glycoprotein Ilb/llla inhibitor, might halt stroke progression by improving flow in the microcirculation. Methods: We conducted a retrospective analysis of patients with subcortical stroke who experienced deterioration and were treated with eptifibatide (loading dose $180 \mu \mathrm{g} / \mathrm{kg}$; infusion $2 \mu \mathrm{g} / \mathrm{kg} /$ $\mathrm{min}$ ) for 24-48 h. Oral antiplatelet agents were started $6 \mathrm{~h}$ before discontinuation of eptifibatide. Results: Twenty-four patients with subcortical strokes were treated. The median admission NIHSS score was 5.0, which worsened to 8.5 (motor 5.0) before starting eptifibatide. The median NIHSS score $24 \mathrm{~h}$ after starting eptifibatide was 5.5. At $24 \mathrm{~h}, 42 \%$ had motor NIHSS scores less than or equal to pre-deterioration scores (50\% for total NIHSS), and 50\% had improved at least 1 motor point compared to pre-eptifibatide scores, which was sustained until hospital discharge. At discharge, the median total NIHSS score was 4.5. Ninety-two percent of patients were discharged home or to inpatient rehabilitation. Treatment was stopped early in 1 case due to a platelet drop $<100,000 / \mu \mathrm{l}$. No systemic or intracerebral bleeding occurred. Conclusions: Eptifibatide infusion may be safe in patients with subcortical ischemic strokes. Future studies are needed to test the safety and potential efficacy of this agent in subcortical stroke progression.

Copyright $\odot 2009$ S. Karger AG, Basel
\end{abstract}

\section{Introduction}

The progression of neurological deficits in acute ischemic stroke is estimated to occur in over $20-40 \%$ of patients. Frequency of clinical worsening after hospitalization varies, depending on the mix of stroke patients and their time of entry into the hospital [1]. Progressive stroke is defined as 'a stroke in which the neurological deficit is still increasing in severity or distribution after the patient's admission to the hospital for observation' [2]. Patients with progressive strokes have increased morbidity and mortality as well as worsened functional outcome compared with those who remain stable [2]. In the Harvard Stroke Registry, 95/471 (20\%) of stroke patients progressed after onset, most with lacunar infarcts [3]. In the Lausanne Stroke Registry, among >3,000 patients, clinical worsening after admission occurred in $29 \%$ of all stroke patients and in $662(34 \%)$ of noncardioembolic ischemic stroke patients [4]. Among 350 Japanese patients in another study, 25\% progressed after admission, and worsening in the hospital occurred in $26 \%$ of lacunar stroke patients [5]. In the study by Steinke and Ley [6], $24 \%$ of patients had worsening of motor deficits after hospitalization, and the predominant subtype of stroke was lacunar infarction (23.9\%). While patients with 'lacunar' infarcts may have good outcome [7], studies have demonstrated that neurological deficits progressed after admission in as many as $62 \%$ of patients and corresponded with poor outcome $[4,8,9]$.

\section{KARGER}

(C) 2009 S. Karger AG, Basel

Fax +41613061234 E-Mail karger@karger.ch www.karger.com www.karger.com/ced
Sean I. Savitz, MD

Department of Neurology, Vascular Neurology Program

University of Texas Health Science Center Houston

6431 Fannin, MSB 7.125, Houston, TX 77030 (USA)

Tel. +1 713500 7066, Fax +1 713500 0692,E-Mail Sean.I.Savitz@uth.tmc.edu 
The zone of ischemia in lacunar infarction involves the small arterial vessels supplying the subcortical structures. The most frequent lesion described is microatheromatous branch vessel disease, whereas lipohyalinosis and fibrinoid necrosis are less frequent, and embolism from large vessel disease may also rarely occur [10-15]. Because of the absence of collateral vessels, the infarct usually extends from the site of occlusion through the territory of the affected penetrating artery [15]; however, the size of the ischemic area is variable, depending on the vessel caliber and extent of ramifications [10, 14]. Progression of motor hemiparesis may thus be caused by either stepwise occlusion of the proximal segment of a perforating artery or distal-to-proximal clot propagation with subsequent occlusion of small branches, leading to enlargement of the lacunar infarct and progressive symptoms [6]. Previous studies have shown that patients who progress have severer deficits upon admission and larger final infarct volumes $[5,9,16]$. These data suggest that these patients have larger areas of hypoperfusion and tissue 'at risk' of infarction.

Various strategies have been investigated in the past to halt stroke progression. Trials of anticoagulation with heparin, heparinoids or low molecular weight heparin have shown no effect in treating neurological worsening, including patients with strokes due to small vessel disease $[17,18]$. In a pilot trial of 10 patients with subcortical strokes, volume expansion was associated with a reversal of worsening deficits [19].

In our center, we have tried various treatments to reverse progression in subcortical stroke patients, including induced hypertension and volume expansion. We found that these therapies were frequently associated with cardiovascular side effects and did not predictably reverse or even halt progression [8]. This indicates that elevating blood flow alone to areas of hypoperfusion may not reliably impact flow-limiting arterial lesions, and that additional strategies are needed to arrest deterioration. If stepwise occlusion or propagation of thrombosis of these small perforator vessels and exhaustion of 'penumbra' distally is a major cause of deterioration, then the glycoprotein (GP) IIb-IIIa inhibitor, eptifibatide, may arrest or even reverse deterioration. GP IIb-IIIa inhibitors block platelet aggregation by antagonizing receptors that bind fibrinogen molecules and form bridges between platelets. GP IIb-IIIa inhibitors could allow endogenous tissueplasminogen activator to dissolve thrombi, and prevent growth and re-formation of thrombi by competitive inhibition with fibrinogen. GP IIb-IIIa inhibitors may also promote vasodilatation through nitric-oxide-mediated mechanisms, and therefore could also augment blood flow to brain tissue at risk [20]. GP IIb-IIIa inhibitors are currently used in clinical practice for acute coronary syndromes and percutaneous coronary interventions. The multifactorial effects of eptifibatide on the microcirculation make it an attractive agent compared with heparin to arrest progression in fluctuating patients.

Eptifibatide, along with other GP IIb-IIIa inhibitors, is increasingly being used off-label to treat patients with acute ischemic stroke syndromes. In order to obtain preliminary data on safety, we conducted a retrospective analysis of our patients with subcortical stroke who had neurological deterioration and then received off-label eptifibatide. Our intention was to use these preliminary data to decide if a larger controlled study should be conducted to determine whether eptifibatide can reverse or halt subcortical stroke fluctuation.

\section{Methods}

We conducted a retrospective analysis of patients treated at our stroke center who had an imaging-proven subcortical stroke and deteriorated on the NIHSS while on our inpatient stroke service after hospital admission. These patients were specifically considered for inclusion in a clinical protocol involving the use of eptifibatide for subcortical strokes. Patients received eptifibatide if they met the following inclusion and exclusion criteria:

\section{Inclusion Criteria}

1 Age 18 years or older.

2 Neuroimaging findings consistent with acute subcortical hemispheric or brainstem ischemic stroke in a penetrating vessel territory on MRI or CT. A purely subcortical infarct had to be visualized on DWI or by CT hypodensity consistent with an acute or subacute infarct in the territory of the patient's symptoms.

3 Deterioration in motor NIHSS $\geq 1$ point within $48 \mathrm{~h}$ of symptom onset, and being able to give eptifibatide within $12 \mathrm{~h}$ of onset of worsening/progression.

4 CT or MRI performed within 120 min before starting eptifibatide, showing no bleeding, midline shift, or new nonsubcortical hypodensity.

\section{Exclusion Criteria}

1 Uncontrolled hypertension at time of treatment (systolic BP $>185$ or diastolic $\mathrm{BP}>110 \mathrm{~mm} \mathrm{Hg}$ ) or hypotension (systolic BP $<120$ or diastolic $\mathrm{BP}<50 \mathrm{~mm} \mathrm{Hg}$ ).

2 Brain CT or MRI evidence of acute cortical or cerebellar infarction.

3 Motor NIHSS score $<1$ in all extremities after deterioration, before eptifibatide.

4 Pregnancy (positive pregnancy test).

5 Intracranial surgery or serious head trauma within the last 14 days. 
6 Seizure prior to deterioration that would confuse assessment of stroke severity or progression.

7 Active internal bleeding.

8 History of intracranial neoplasm, arteriovascular malformation, or aneurysm.

9 Known bleeding disorders, including international normalized ratio $>1.7$, platelet count $<100,000 \mathrm{~mm}^{3}$ and unexplained hematocrit count $<30 \%$.

10 Serum glucose $>400$ or $<50 \mathrm{mg} / \mathrm{dl}$.

11 Patients with end-stage renal disease requiring dialysis.

12 Patients with other likely cause of deterioration, such as metabolic abnormalities, fever, hypoxia, congestive heart failure, or hypotension.

13 LOC $>1$ on 1a NIHSS score.

14 Symptoms due to a new stroke in a different vascular territory (new stroke).

15 Receiving anticoagulation with heparin for venous thrombosis, cardioembolic source, or other medical conditions (antiplatelet therapy and subcutaneous heparin or low molecular weight heparin for deep vein thrombosis prophylaxis was allowed).

Patients were screened by a member of the stroke team. Initial clinical assessment included vital signs, blood glucose, complete blood counts, coagulation profile, and baseline NIHSS. Patients already treated with rt-PA for their stroke were included. Patients had a CT (noncontrast) or MRI of the brain prior to treatment. Patients or legal next-of-kin provided informed consent.

All patients were managed by the same stroke team and monitored in the stroke unit or the neurocritical care unit (when necessary) for close observation. Patients received standard of care with regard to deep venous thrombosis prophylaxis. Euvolemia was maintained with normal saline infusion based on the clinical examination (invasive monitoring was not routinely used). Hyperglycemia was treated aggressively with sliding-scale insulin to maintain normal blood glucose. All patients had routine stroke workups, including echocardiography, carotid imaging, electrocardiogram, and risk factor stratification. Patients received standard nursing care and rehabilitation therapies.

Deterioration in neurological status was defined as an increase of 1 point or greater in the motor portion of the NIHSS for assessment of extremity movement ( $0=$ no drift, $1=$ drift, $2=$ some effort vs. gravity, 3 = no effort vs. gravity, $4=$ no movement). Worsening in facial paresis or articulation may have occurred, but was not included in the measurement of the motor score on the NIHSS examination.

As standard of care, the head of the bed was placed at zero degrees to the horizon, and emergent brain imaging was performed. Euvolemia was assured by normal saline IV based on the clinical examination (invasive monitoring was not routinely used). Creatinine, hemoglobin, and platelet measurements must have been performed within $24 \mathrm{~h}$ of treatment. Eptifibatide $(180 \mu \mathrm{g} / \mathrm{kg}$ bolus followed by $2 \mu \mathrm{g} / \mathrm{kg} / \mathrm{min} ; 1 \mu \mathrm{g} / \mathrm{kg} / \mathrm{min}$ for patients treated within $24 \mathrm{~h}$ of IV rt-PA) was administered intravenously for 24-48 h. Hemoglobin and platelets were monitored every $6 \mathrm{~h}$, and blood pressure and neurological assessment were performed every hour during the infusion. The infusion was stopped early for a drop in hemoglobin ( $\mathrm{Hb})$ of at least $3 \mathrm{~g} / \mathrm{dl}$, drop in platelets to $<100,000 /$ $\mu l$, clinically significant bleeding, or intracerebral hemorrhage. Brain CT/MRI was repeated as clinically indicated. Anticoagulation with full-dose heparin, Lovenox, or warfarin was not used
Table 1. Patient characteristics $(\mathrm{n}=24)$

\begin{tabular}{lc}
\hline Age (mean \pm SD), years & $63 \pm 15$ \\
Female, \% & 54 \\
Ethnicity, \% & \\
$\quad$ Black & 50 \\
$\quad$ White & 42 \\
$\quad$ Hispanic & 8 \\
History of hypertension, \% & 83 \\
History of diabetes, \% & 42 \\
History of prior TIA, \% & 8 \\
Parent vessel involved, \% & \\
$\quad$ Middle cerebral artery & 54 \\
$\quad$ Basilar artery & 42 \\
$\quad$ Vertebral artery & 4 \\
Baseline NIHSS score (median) & 5 \\
Time from stroke onset to treatment & \\
$\quad$ with eptifibatide (mean \pm SD), h & $36 \pm 22$ \\
Length of stay (median and range), days & $5(2-11)$ \\
\hline
\end{tabular}

during the infusion of eptifibatide. Antiplatelet agents were started $6 \mathrm{~h}$ before stopping the eptifibatide infusion.

Assessments of the NIHSS score were performed at 24 and $48 \mathrm{~h}$ after the start of the eptifibatide infusion and on the day of discharge by a stroke team member. If the NIHSS was not recorded, it was abstracted from the patient's medical record. We also tracked disposition and discharge modified Rankin Scale scores. The off-label use of eptifibatide has been internally monitored for patient safety at our center.

Patients who met inclusion criteria were collected to address the following questions:

1 Is eptifibatide (bolus $180 \mu \mathrm{g} / \mathrm{kg}$ followed by infusion of $2 \mu \mathrm{g} /$ $\mathrm{kg} / \mathrm{min}$ over $24 \mathrm{~h}$ ) safe to give within $12 \mathrm{~h}$ of deterioration in patients with acute subcortical ischemic strokes? The primary outcome was serious systemic bleeding (defined as clinical bleeding or drop in $\mathrm{Hb}>3 \mathrm{~g} / \mathrm{dl}$ or necessitating transfusion) or symptomatic intracranial hemorrhage or parenchymal hemorrhage (PH-2). Safety was defined as less than $10 \%$ incidence.

2 What is the incidence of improvement in the patients' motor component of the NIH stroke scale (NIHSS) $24 \mathrm{~h}$ after eptifibatide treatment compared to the baseline motor NIHSS score prior to the deterioration? This was considered a secondary outcome measure.

3 What is the incidence of improvement in the patients' motor NIHSS score by at least 1 point $24 \mathrm{~h}$ after eptifibatide treatment compared to the NIHSS score at the time of deterioration? This was an additional secondary outcome measure.

\section{Results}

Twenty-four patients were treated for deteriorating acute subcortical ischemic stroke. The average age was 63 years (SD 15 years); $54 \%$ were female; $50 \%$ were black and $42 \%$ were white (table 1). Half of the patients (56\%) had 


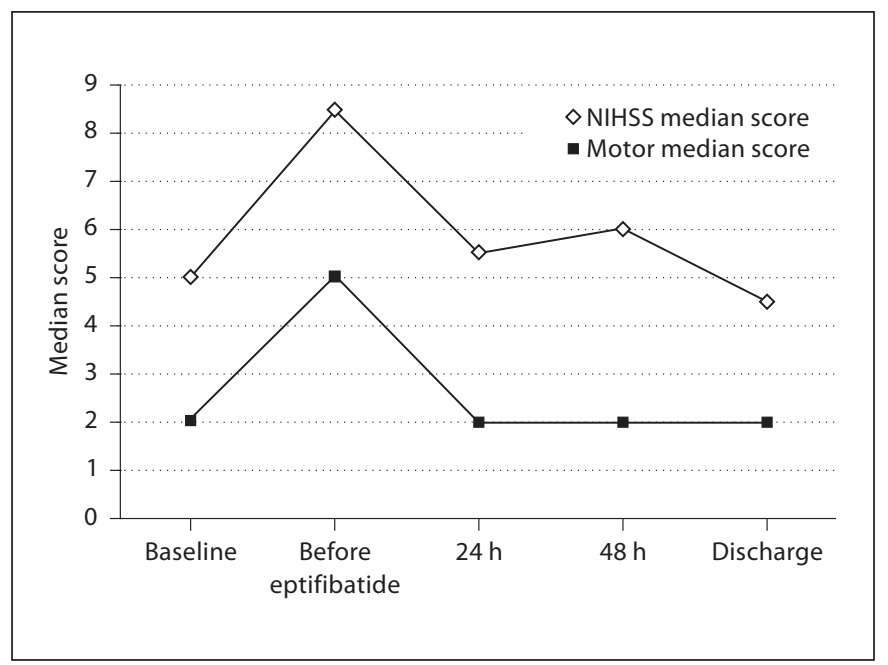

Fig. 1. Changes in total and motor NIHSS scores from admission (baseline) to hospital discharge.

Table 2. Percentage of patients who experienced improvements in NIHSS scores after treatment with eptifibatide

Patients

$\%$

Score at $24 \mathrm{~h}$ after eptifibatide $\leq$ admission score

Motor component $\quad 42$

Total Score

Score at $24 \mathrm{~h}$ after eptifibatide $\leq$ pre-eptifibatide score Motor component

50

Total

67

Hospital discharge score $\leq$ hospital admission score

Motor

37.5

Total

50

Hospital discharge score < pre-eptifibatide score

Motor

Total
50

71

strokes within the deep structures fed by perforators of the middle cerebral artery, while the other half had strokes in the brainstem fed by perforators of a vertebral artery or basilar artery. No patient had a hemorrhage on baseline imaging. Five of the patients (21\%) had moderate-tosevere stenosis of the parent artery on CT or MR angiography. Six patients (25\%) had received IV tPA a mean of $11.3 \mathrm{~h}$ prior to starting eptifibatide.

The median baseline NIHSS was 5.0, which worsened to 8.5 before treatment with eptifibatide (fig. 1). Motor scores worsened from a median of 2.0 to 5.0. Patients were treated a mean of $36 \mathrm{~h}$ after initial stroke symptoms,

within $12 \mathrm{~h}$ of deterioration, and were treated with eptifibatide for a mean of $33.5 \mathrm{~h}$. Treatment was stopped early in only 1 case due to a decline in platelet count to $<100,000 / \mu l$. No systemic or symptomatic intracerebral bleeding occurred. No patient had a decline in hemoglobin by more than $3 \mathrm{~g} / \mathrm{dl}$. Only 1 patient had hemorrhagic infarction, and this was only grade 1 . All patients received oral antiplatelets before discontinuation of eptifibatide.

The median motor and total NIHSS scores at $24 \mathrm{~h}$ after eptifibatide infusion were 2.0 and 5.5, respectively (fig. 1). Ten patients $(42 \%)$ returned to their pre-deterioration (baseline) motor NIHSS score, and 50\% returned to their pre-deterioration total NIHSS score by $24 \mathrm{~h}$ after eptifibatide was administered (table 2). Half of the patients improved by at least 1 point on the motor portion of the NIHSS by $24 \mathrm{~h}$ compared to their pre-eptifibatide score (defined as NIHSS collected right before the drug was administered). This improvement persisted through the time of discharge. By discharge, 37.5\% of patients had motor NIHSS scores that were less than or equal to baseline scores at hospital admission. Five patients stabilized and had no change in motor NIHSS scores when comparing the pre-eptifibatide score with that of the hospital discharge score. Seventeen patients (71\%) had improvement in total NIHSS by discharge compared to the pre-eptifibatide NIHSS. Eleven (46\%) patients had lower total NIHSS scores at discharge compared with baseline, before the deterioration. Two patients continued to deteriorate, and had a discharge NIHSS score 1 point higher than that recorded before treatment with eptifibatide. At discharge, the median total and motor NIHSS scores were 4.5 and 2.0, respectively. All but 2 patients (92\%) were discharged home or to inpatient rehabilitation. The median discharge mRS was 3.0.

\section{Discussion}

Deterioration after subcortical stroke is common. There are no proven treatments to abort or reverse deterioration once it begins. Treatment with high-potency antiplatelets may prevent clot propagation and occlusion of small penetrating vessels, thereby halting stroke progression. This case series of patients with deteriorating subcortical strokes demonstrated that eptifibatide may be safe in this patient population. There was no evidence of bleeding or other serious adverse effects of 1-2 days of eptifibatide treatment at the doses used, including those patients who received IV tPA. In general, the percentage of patients with subcortical stroke who deteriorate and 
then return to pre-deterioration motor function by $24 \mathrm{~h}$ has not been well-characterized. Thirty-eight percent of our treated patients achieved this goal. In addition, roughly half of our patients did improve at least 1 motor point by $24 \mathrm{~h}$, and also improved to better than baseline NIHSS scores by the time of discharge. Although this study was not designed to test the efficacy of eptifibatide treatment, these results, coupled with the very low adverse event rate, are encouraging and justify further study of eptifibatide in this setting.

Eptifibatide is being evaluated for other cerebrovascular conditions besides subcortical strokes. In the CLEAR trial, it was tested as an adjunct to IV tPA [21], and there have been reports of its use to augment recanalization and prevent re-occlusion after intra-arterial thrombolysis [22]. However, the present study focuses on a different stroke population. Subcortical strokes may be a safe application of delayed GP IIb-IIIa therapy since the infarcts are smaller and less likely to bleed.

Another question of interest is the relationship between the time of initial symptom onset and the time between deterioration and the start of eptifibatide infusion (0-3 vs. 3-12 h). These intervals, and their relation to partial and complete response rates, could not be examined in this case series. Not only were the number of patients enrolled too small for such a subanalysis, but the exact timing of deterioration was uncertain in our patients. Previous data from our center [8] showed that deterioration occurred on average a full day after symptom onset, and often in the second and even third days, consistent with prior studies $[23,24]$. In this case series, eptifibatide was started on average $36 \mathrm{~h}$ after symptom onset and $12 \mathrm{~h}$ after deterioration was first noted. However, since this protocol did not involve observation of all subcortical stroke patients, and only included patients after they had deteriorated, our data on the incidence and timing of deterioration is inexact. The issue of timing of eptifibatide infusion will therefore have to be explored in a subsequent study.

We had no control group, and therefore we cannot assume that the improvements in neurological function were due to eptifibatide rather than the natural history of the patient's disease. We believe that our initial pilot data, however, provide evidence that eptifibatide is safe in this stroke population and support the continued investigation of this drug for progressing subcortical stroke, for which there are no effective therapies to arrest neurological worsening. We are now characterizing the natural history of small vessel fluctuation in patients with subcortical strokes. This information will help us in planning a future study to test whether eptifibatide can reverse deterioration in progressing subcortical stroke. Our ultimate goal is to assess whether recovery to pre-deterioration deficits is increased in patients treated with eptifibatide compared with placebo-controlled patients.

\section{Acknowledgements}

S.M.-S., A.D.B., H.H., H.S. and A.T.A. were supported by the training grant 5-T32-NS007412-09 from the National Institutes of Health to the University of Texas-Houston Medical School Stroke Program. S.I.S. was supported by AHA 0475008N.

\section{References}

1 Caplan LR: Worsening in ischemic stroke patients: is it time for a new strategy? Stroke 2002;33:1443-1445.

-2 Davalos A, Cendra E, Teruel J, Martinez M, Genis D: Deteriorating ischemic stroke: risk factors and prognosis. Neurology 1990;40: 1865-1869.

-3 Mohr JP, Caplan LR, Melski JW, Goldstein RJ, Duncan GW, Kistler JP, Pessin MS, Bleich HL: The Harvard Cooperative Stroke Registry: a prospective registry. Neurology 1978; 28:754-762.

4 Yamamoto H, Bogousslavsky J, van Melle G: Different predictors of neurological worsening in different causes of stroke. Arch Neurol 1998;55:481-486
5 Tei H, Uchiyama S, Ohara K, Kobayashi M, Uchiyama Y, Fukuzawa M: Deteriorating ischemic stroke in 4 clinical categories classified by the Oxfordshire community stroke project. Stroke 2000;31:2049-2054.

6 6 Steinke W, Ley SC: Lacunar stroke is the major cause of progressive motor deficits. Stroke 2002;33:1510-1516.

7 Libman RB, Sacco RL, Shi T, Tatemichi TK, Mohr JP: Neurologic improvement in pure motor hemiparesis: implications for clinical trials. Neurology 1992;42:1713-1716.

8 Labiche LA, Moye L, Campbell M, Grotta JC: Progression of subcortical infarcts. Abstract 8th Int Symp Thrombolysis Acute Stroke Ther, Whistler, 2004

-9 Nakamura K, Saku Y, Ibayashi S, Fujishima M: Progressive motor deficits in lacunar infarction. Neurology 1999;52:29-33.
10 Challa VR, Bell MA, Moody DM: A combined hematoxylin-eosin, alkaline phosphatase and high-resolution microradiographic study of lacunes. Clin Neuropathol 1990;9: 196-204.

11 Fisher CM: The arterial lesions underlying lacunes. Acta Neuropathology 1969;12:115.

12 Fisher CM: Capsular infarcts: the underlying vascular lesions. Arch Neurol 1979;36:6573.

$\checkmark 13$ Fisher CM, Curry HB: Pure motor hemiplegia. Trans Am Neurol Assoc 1964;89:94-97.

14 Marinkovic SV, Milisavljevic MM, Kovacevic MS, Stevic ZD: Perforating branches of the middle cerebral artery: microanatomy and clinical significance of their intracerebral segments. Stroke 1985;16:1022-1029. 
15 Mohr JP, Marti-Vilalta JL: Lacunes; in Barnett HJM, Mohr JP, Stein BM, Yatsu FM (eds): Stroke: Pathophysiology, Diagnosis, and Management. New York, Churchill Livingstone, 1998, pp 599-622.

16 Lodder J, Gorsselink EL: Progressive stroke caused by CT-verified small deep infarcts; relation with the size of the infarct and clinical outcome. Acta Neurol Scand 1985;71: 328-330.

- 17 Adams HP Jr, Bendixen BH, Kappelle LJ, Biller J, Love BB, Gordon DL, Marsh EE, 3rd: Classification of subtype of acute ischemic stroke: definitions for use in a multicenter clinical trial. TOAST. Trial of ORG 10172 in acute stroke treatment. Stroke 1993;24:3541.
18 Low molecular weight heparinoid, ORG 10172 (danaparoid), and outcome after acute ischemic stroke: a randomized controlled trial. The Publications Committee for the Trial of ORG 10172 in Acute Stroke Treatment (Toast) Investigators. JAMA 1998;279: 1265-1272.

19 Frey J: Hemodilution therapy for lacunar stroke: treatment results in 10 consecutive cases. J Stroke Cerebrovasc Dis 1992;2:136145.

20 Heitzer T, Ollmann I, Koke K, Meinertz T, Munzel T: Platelet glycoprotein IIb/IIIa receptor blockade improves vascular nitric oxide bioavailability in patients with coronary artery disease. Circulation 2003;108:536541.

-21 Pancioli AM, Broderick J, Brott T, Tomsick T, Khoury J, Bean J, del Zoppo G, Kleindorfer D, Woo D, Khatri P, Castaldo J, Frey J, Gebel J Jr, Kasner S, Kidwell C, Kwiatkowski T, Libman R, Mackenzie R, Scott P, Starkman S, Thurman RJ; CLEAR Trial Investigators: The combined approach to lysis utilizing eptifibatide and rt-PA in acute ischemic stroke: the CLEAR stroke trial. Stroke 2008;39:3268-3276.
22 Qureshi AI, Hussein HM, Janjua N, HarrisLane P, Ezzeddine MA: Postprocedure intravenous eptifibatide following intra-arterial reteplase in patients with acute ischemic stroke. J Neuroimaging 2008;18:50-55.

23 Kitanaka C, Teraoka A: Clinical features of progressive lacunar infarction - retrospective analysis of patients with motor syndromes. Neurol Med Chir (Tokyo) 1995;35: 663-666.

-24 Jorgensen HS, Nakayama H, Raaschou HO, Olsen TS: Effect of blood pressure and diabetes on stroke in progression. Lancet 1994; 344:156-159. 